\title{
Research on digital exhibition Old Summer Palace somatosensory interaction based on Leap Motion
}

\author{
Liu Dianxing \\ BeiJing Tsinghua TongHeng Urban Planning \& Design Institute, Beijing China, 100085
}

Keywords: Virtual reality; Leap Motion; Somatosensory Interaction

\begin{abstract}
The ancient site of the real return is a new research field in digital cultural heritage protection field, using virtual reality technology to reproduce the ancient ruins can be completely without changing the original appearance of the site, and retain the modification to reconstruct the landscape, can solve the reconstruction of good and protection of the contradiction, also need not worry about historical data missing caused irreparable regret. Based on the national important cultural heritage "Old Summer Palace" digital display as the main background, in order to enhance the perception of the whole scene details of real people, in the traditional virtual exhibition platform to design a Leap Motion interaction module, display a kind of non-contact test gesture interaction techniques, so that people can interact operating through different gestures of different objects and components in the scene, make it real in finite basis does not contact the real object of the understanding of "Old Summer Palace", and reduce the dependence on the mouse and keyboard and control complexity, played down the boundary of virtual scene and the real environment. Finally through the virtual scene high fidelity tested the utility of somatosensory interaction technology in the virtual display system.
\end{abstract}

\section{Introduction}

How to improve the display effect of ancient ruins, is a subject of archaeological planning architectural design and tourism professional workers have been exploring. In recent years, a variety of new information display and interaction devices appeared in people's field of vision, the development of equipment and technology at the same time, the new application demand has been showing also emerge, people are no longer satisfied with playing information passive recipient role, and hope to further play the creation of information the participants. How to use the advanced equipment and technology, to meet the needs of people showing the process of interaction, is currently a hot research field. As the Microsoft Corp founder Bill Gates in Las Vegas in 2008 international consumer electronics show that, people interact with computers will have a great change, gradually be more humane, more intuitive means of science and technology in place of, various high-definition display technology will be more easy to be used, can let more people connected to experience.

In China, almost the sites display mode is currently still in the authenticity protection display or static simulation display etc. These show the way is very good protection for the site, but there are ornamental participation is not strong, the form of a single, interactive poor defects, not well mobilized the site visit participants viewing interest. Therefore, to explore the efficient way of interactive experience site, improve the site exhibits in the visitor participation and interaction is one of the core problems of degree of site protection and display to be solved at the present stage. This paper will be an important large site, China's World Heritage - Old Summer Palace for research and application demonstration object, focus on site interaction experience problems. Scientific interpretation of site cultural connotation, comprehensively revealed the site value, improve the participation degree of the public key, reflect the site protection and utilization, the study on the efficient display of large site, has important significance to promote the development of cultural industry sites. 


\section{Leap Motion}

Leap Motion (Figure 1) since 2013 America community of science and technology is the most popular one of the somatosensory equipment, It allows the user through the most simple gesture let between human and computer interact, and created the body feeling technology based on the Airspace app store, Mainly involved in somatosensory and interaction. The popularity of the worthy and comparable iPhone launch.

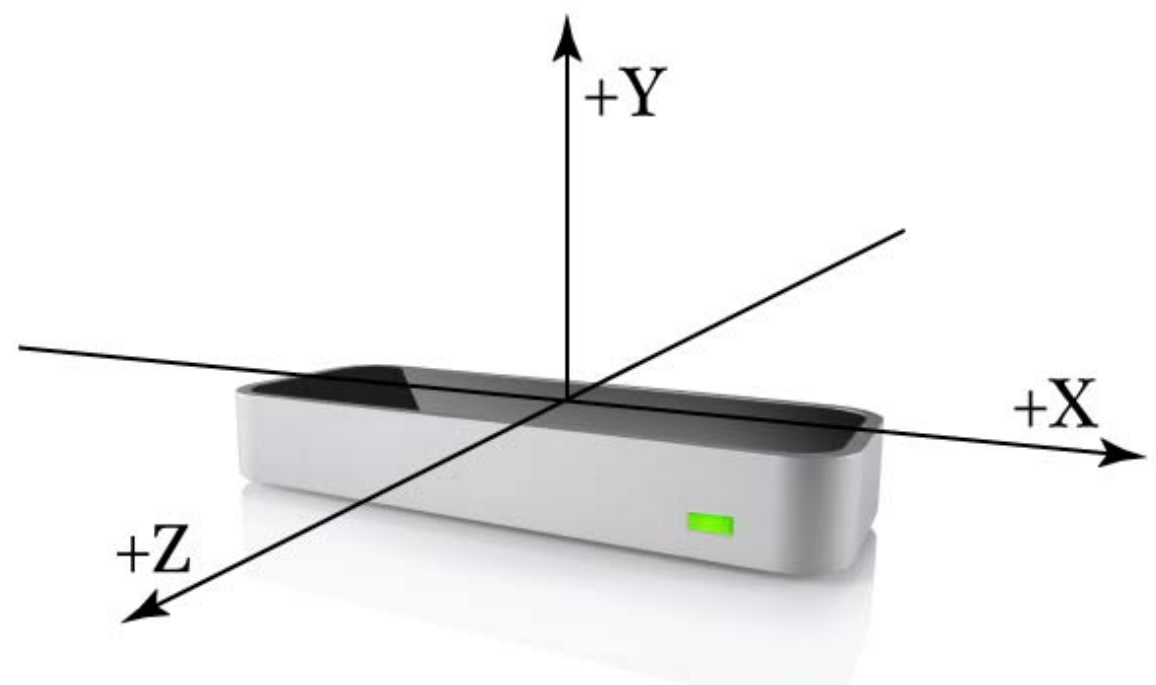

Figure 1

According to the technical publicity official, in addition to the somatosensory control in front of the computer, Leap Motion has also begun to affect some of the traditional industries, as a sculptor, architect can use this technology to complete a virtual works, and then through the printer to print out the model of 3D.

By studying the research thinks, gesture manipulation from the perspective of Leap Motion concept, based on gestures, suitable for desktop near distance, for intelligent interaction somatosensory display mode. If you can Leap Motion the gestures used in display of ancient sites, will be a new research field site protection.

\section{Design and Implementation}

Using the real, computer software and peripherals based on a combination of interactive display mode, to display as the foundation, the physical and electronic apparatus, computer program, interactive mechanism combined, so as to give the participants to the sense of reality, at the same time, but also solves the traditional exhibition in the background, connotation, historical relics, artifacts related to tectonic process or problem to be difficult to introduce large blocks of text presentation. Introduction video, analog artifacts manufacturing process by way of participants trigger, increasing interest and explain the participation of the participants of cultural relics. But this kind of interactive display mode, and also increase the degree of freedom of participants in the tour process, so that participants can selectively through its own initiative participation. In the process of the contact, explore and discover, enhancement of the sites of cultural understanding.

\section{Framework}

In this paper, the design of human-computer interaction applications, namely building ancient pavilion, the pavilion on the basis of mortise and tenon structure is split into several modules, users through the program prompts and equipment Leap Motion complete gutting the building. In the program frame, abandon interactive way in the past of the mouse and keyboard, with the aid of Leap Motion into complete man-machine interface traditional users, and by the gesture into the scene, and the model is set up, finally finish the task over the entire program. From the embodiment can also reflect the Leap Motion is not only a piece of equipment, but also is a kind of new 
computer control concept, its core is the desktop close gestures.

\section{Model}

Mortise and tenon structure, is the wood frame structure as the structure, the main structure, composed of column, beam, Shun purlin from the construction of major components, each component of the junction between the coincide with tenon and mortise, constitutes a flexible framework. We selected the more common Old Summer Palace Pavilion modeling, as a core component of the whole scene. Participants randomly crawls scene each model can be obtained by Leap Motion, and random combinations, until eventually merged into a complete ancient pavilion.
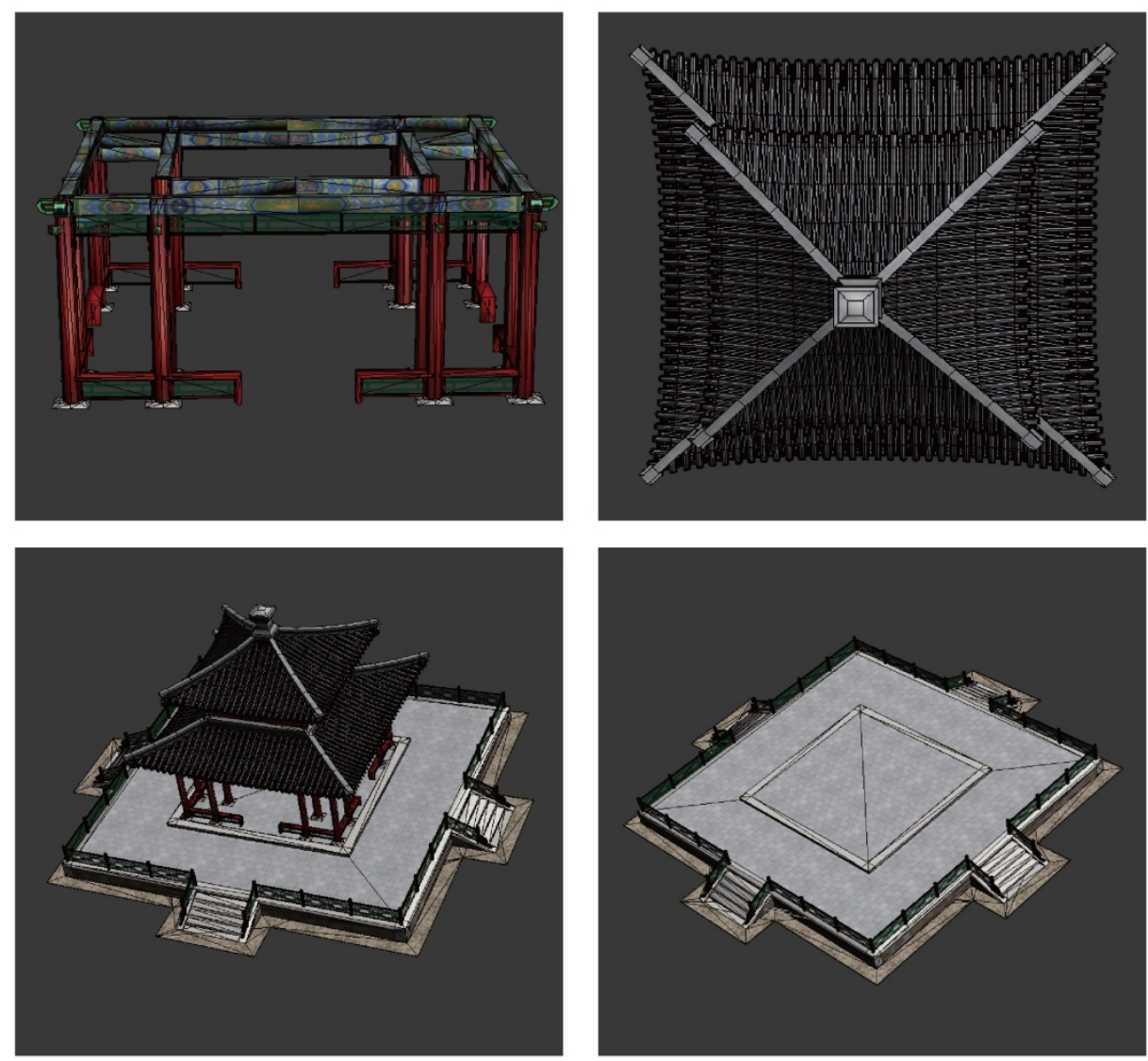

Figure 2

\section{Interaction}

The current Leap Motion provided by SDK (Software Development Kit, a software development kit) contains the finger and tool tracking, gesture definition and recognition, palm movement and support for third party plug-ins embedded functions, support for selecting, moving, rotating, zoom, drag and drop interaction by direction and position data, therefore also allows the program designers play creative design unconstrained have the personality of game interaction.

At the welcome screen, users can swipe left to the palm of your hand, and you are prompted to exit, if exit virtual point screen; right waving palm is skipped welcome screen, enter the start screen. At the beginning of the screen, the users can virtual point screen or right waving palm into model ready for screen, also can be left waving palm returned welcome screen. 


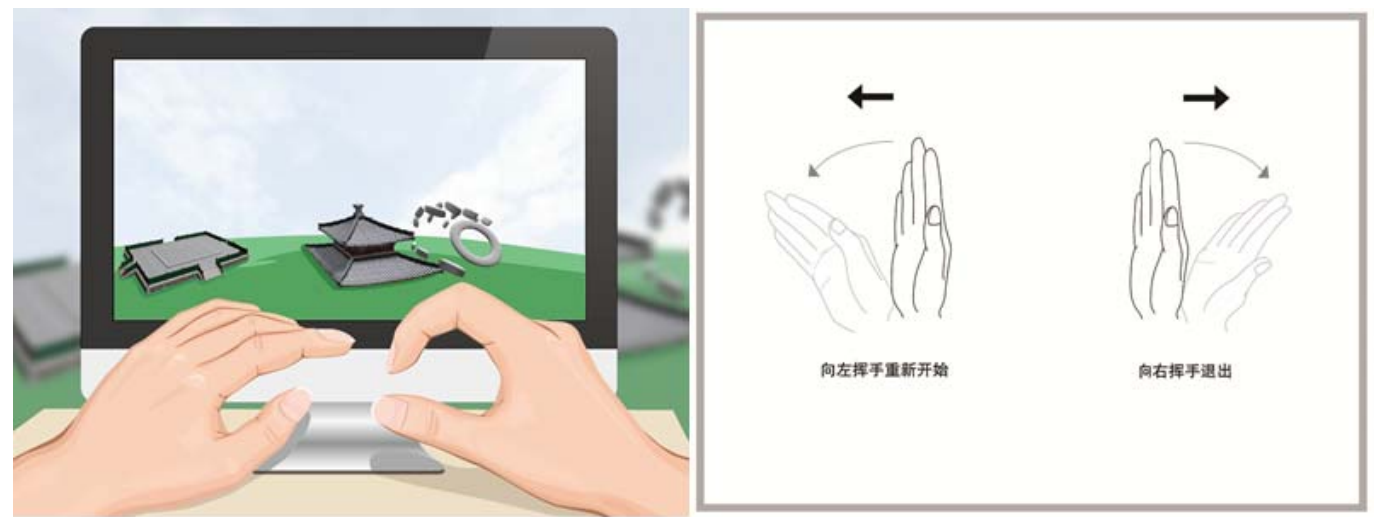

Figure 3

In the model ready for screen, users need to wait 10 seconds, will automatically enter the model assembly screen. In the model of assembly screen, model from the air disintegration scattered in the scene, the user can grab model and complete the building, and in the upper left corner of the screen with an auxiliary top, can be opened or closed by pressing the $\mathrm{H}$ key.

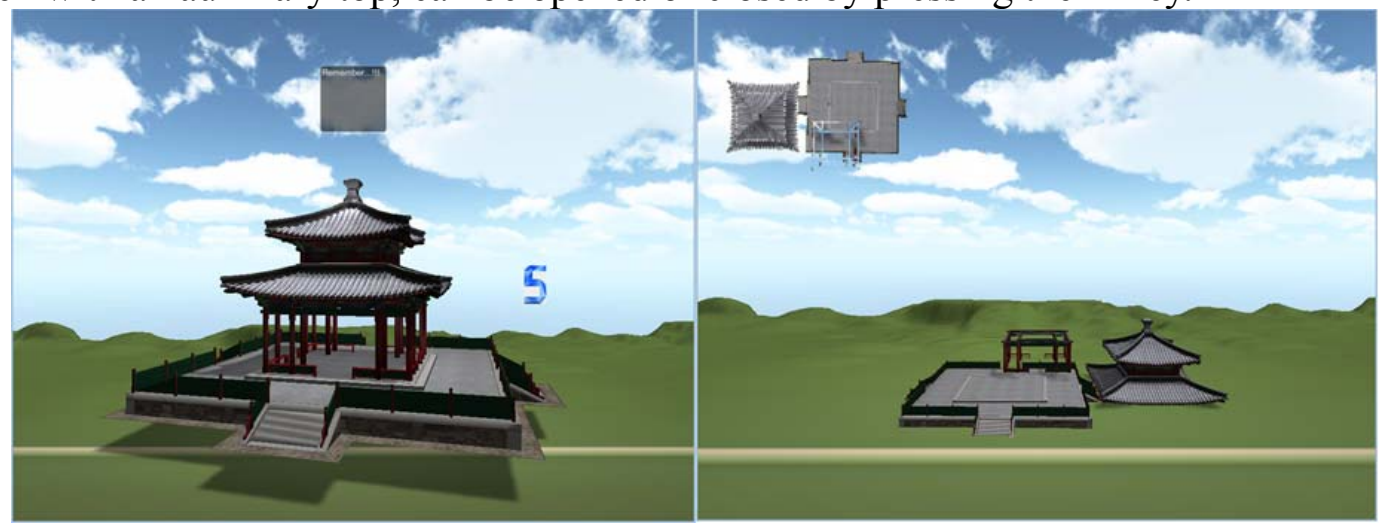

Figure 4

If the user successfully completed will smoothly into the end of the screen, through to the left or right palm start waving, waving palm quit the program.

\section{Display}

In the interactive process, hand data users completely relies on the Leap provided by Motion to complete the relevant operation, abandons the traditional mouse and keyboard, reflects the precision performance of modern interactive devices have developed rapidly, and the related software technology, it brings very interesting experience for users.

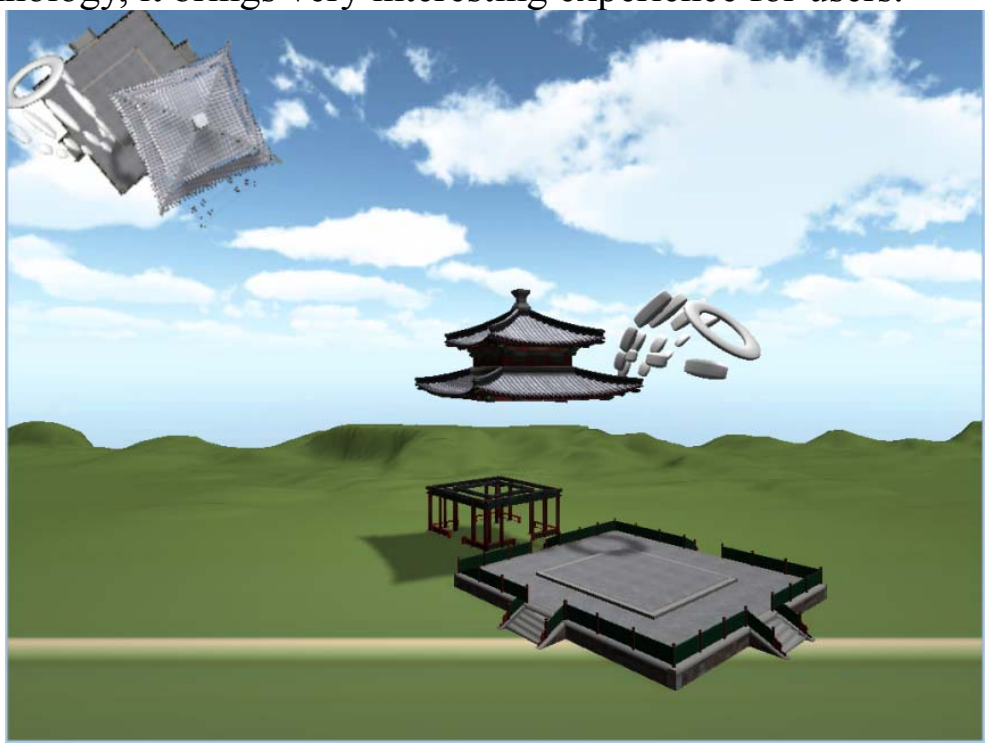

Figure 5 
The use of Leap Motion that somatosensory interaction device, can not only meet the visitors on the ancient building touch curious psychology, and can prolong the using life of the facilities, to reduce the venue of the repair and management costs.

Leap Motion CEO Mike Barker Ward said, now between the computer and the user of the existence of obstacles, the best way to bridge the gap is to use "interactive ability of each person to computer the most natural". Leap Motion provides a direction for human and computer interactive mode change, it deeply into any place with computer, will appear in the medical, financial, engineering, game and many other areas, by waving, pinch, wipe gestures to control the interaction.

\section{Conclusion}

With the development of the current digital interactive technology, digital interactive means solid cultural sites can also try the multi-directional, touch speech somatosensory interactive gives people a more interactive experience. For example, using virtual reality technology, the new, such as helmets, glasses, depth camera interaction devices and stereo display technology, visitors can good experience to visit the fun: immersion, interactivity of virtual environment experience etc. Similar to the Leap Motion through the somatosensory equipment visitors can freely grasping a work of art, or even feel it shape weight and texture, and other visitors in the virtual space double exchange. This kind of digital display platform pays more attention to the actual experience and sense of fun interaction, but the implementation of this kind of experience to display technology is more complex, often need to virtual reality of high-end technology and equipment can be realized; sometimes by site environmental constraints, also need a long time to develop and popularize.

Whether it is pure virtual ancient ruins, the ruins of the entity or the digital display, has the extremely broad development prospect especially in the context of globalization and networking environment, people on the network information is not subject to regional time limited access demand grow with each passing day, although the virtual scene can not completely replace the real display effect, but in the network interaction information transfer and information sharing is not limited by space-time has unique function entity museum do not possess, Leap Motion also provides a very good network adaptability, but its unique business value has already caused the extensive concern.

\section{References}

[1] dong-hai zhang. Sports technical analysis of $3 \mathrm{~d}$ reconstruction framework of computer automatic recognition [J]. Science and technology, 2013, (6) : 146-148.

[2] Han Cheng gui-he qin, palace. Become a three-dimensional reconstruction method based on structure light color [J]. Journal of jilin university: engineering science, 2013 lancet (5) : 1343-1351.

[3] min wang you-ren wang, zhang Zhai. Three-dimensional structure reconfigurable array online since diagnosis and fault tolerance method [J]. Journal of instruments and meters, 2013, (3) : 650-656.

[4] Wang Zhongren yue-bin zhou,. Establish resplendence ardently based on line structured light and movement platform of three dimensional reconstruction method [J]. Journal of tool technology, 2015, (1) : 81-83.

[5] peng-fei Lin, Deng Tianmin, li Yang. 3 d reconstruction based on point cloud track foreign body detection system research [J]. Science advisory, 2014, (27) : 69-71. 